\title{
Interaction between a large buoyant bubble and turbulence
}

\author{
Aurore Loisy and Aurore Naso* \\ Laboratoire de Mécanique des Fluides et d'Acoustique, CNRS, École Centrale de Lyon, \\ Université Claude Bernard Lyon 1, and INSA de Lyon, 36 Avenue Guy de Collongue, \\ F-69134 Écully Cedex, France \\ (Received 18 July 2016; published 25 January 2017)
}

\begin{abstract}
The free rise of isolated, deformable, finite-size bubbles in otherwise homogeneous isotropic turbulence is investigated by direct numerical simulation. The Navier-Stokes equations are solved in both phases subject to the pertinent velocity and stress conditions at the deformable gas-liquid interface. The bubble rise velocity is found to be drastically reduced by turbulence, as is widely known for microbubbles. The probability distribution functions of the horizontal bubble acceleration component are well fitted by a log-normal distribution. The distributions of the vertical components are negatively skewed, a property related to the fact that bubbles experience on average stronger decelerations than accelerations. An assessment of the correlations of bubble acceleration with properties of the surrounding flow is used to define estimates of the liquid velocity and vorticity entering in liquid acceleration and lift forces. Finally, fast rising bubbles are found to preferentially sample downflow regions of the flow, whereas those subjected to a higher turbulence level have an increased residence time in swirling regions, some features similar to those of small bubbles.
\end{abstract}

DOI: 10.1103/PhysRevFluids.2.014606

\section{INTRODUCTION}

The ability to describe the behavior of turbulent bubbly flows is crucial for the design and operation of industrial equipment for a wide range of applications (e.g., oil or gas transport, nuclear reactors, $\mathrm{CO}_{2}$ capture). The complexity of the coupling of turbulence and multiphase flow is a formidable challenge and one is bound to rely on empirical correlations to predict the behavior of such systems.

The trajectories of bubbles that are smaller than the smallest length scales of the flow can be computed in a Lagrangian manner from the integration of an explicit equation of motion and their action on the surrounding flow can be modeled by point forces acting on the carrier phase. This approach has been extensively used to investigate the dynamics of microbubbles in three-dimensional homogeneous isotropic turbulence and their backreaction on the surrounding flow [1-6]. These studies highlighted the crucial role played by the lift force in retarding the rise of small bubbles and on the modulation of turbulence by their presence.

However, in many situations of practical interest, the characteristic size of the bubbles is in the inertial range of scales. In that case, the bubble dynamics cannot be accurately captured by standard point-bubble models [7]. To properly capture the physics of turbulent flows laden with finite-size bubbles in a numerical simulation, all the scales of the carrier flow and of the disturbances induced by their motion would have to be resolved, in the current absence of accurate simplified models.

The dynamics of finite-size particulates in a turbulent environment has been studied experimentally, but primarily for solid particles [8-13]. Experiments on bubbles have also been conducted recently [14,15], but the measurement of the carrier-phase velocity field in the immediate vicinity of the particulates remains a difficult task. It is therefore of interest to investigate whether fully resolved numerical simulations can usefully complement laboratory experiments.

While the simulation of the interaction between isotropic turbulence and large solid spherical particles has been recently performed in increasingly complex configurations (fixed particle [16],

\footnotetext{
*Corresponding author: aurore.naso@ec-lyon.fr
} 
free nonbuoyant particle [17,18], and settling particles [19]), the case of clean bubbles still remains largely uncharted territory: The state of the art amounts to the early large-eddy simulations of [20,21], which considered a large bubble with imposed spherical shape held fixed on the axis of a weakly turbulent pipe flow. Direct numerical simulations of turbulent bubbly flows with well-resolved gas-liquid interfaces are rather commonly performed in highly inhomogeneous situations (vertical channels) (see, e.g., [22-25]). Compared to those of solid particles, direct numerical simulations of bubbly flows are even more challenging because internal gas circulation and interface deformation need to be accounted for, which in turn requires solving in both phases the Navier-Stokes equations subject to the pertinent conditions at the deformable moving interface.

We present in this paper the results of direct numerical simulations (DNSs) of a single, deformable, finite-size bubble freely rising in an otherwise homogeneous isotropic turbulent flow. In the present investigation the turbulence intensity, defined as the ratio between the root mean square of velocity in a one-phase flow and the bubble rise velocity in a liquid at rest, is of order one. The methodology is first described in Sec. II. Results are presented in Sec. III: The deformation, velocity, and acceleration statistics of a large bubble rising in a turbulent environment are first characterized (Sec. III A), followed by an investigation of whether the bubble acceleration is correlated to appropriately defined liquid flow properties (Sec. III B), after which the liquid flow sampled by the bubble is investigated (Sec. III C). A summary is given in Sec. IV.

\section{METHODOLOGY}

\section{A. Physical parameters}

We investigate in this paper the statistically stationary rise of a single buoyant bubble in an otherwise homogeneous isotropic turbulent liquid flow. The primary dimensionless parameter characterizing the interaction between buoyant bubbles and turbulence is the turbulence intensity $\beta=u_{0} / V_{T}$ [1-6], where $u_{0}$ is the root mean square of the liquid velocity fluctuations in the absence of the bubble and $V_{T}$ is the terminal bubble velocity in still liquid. In the present study, $\beta$ is $O(1)$ and is modified through $V_{T}$ as explained in the next paragraph. The bubble characteristic size $d_{b}$, defined as the diameter of the volume-equivalent sphere, is equal to the Taylor microscale $\lambda=\sqrt{15 v u_{0}^{2} / \varepsilon_{0}}$, where $v$ is the kinematic viscosity of the liquid and $\varepsilon_{0}$ is the mean dissipation rate per unit mass of the single-phase flow. The ratios between $d_{b}$ and the turbulence length scales (Kolmogorov scale $\eta=\left(v^{3} / \varepsilon_{0}\right)^{1 / 4}$, Taylor scale $\lambda$, and integral scale $\left.L=u_{0}^{3} / \varepsilon_{0}\right)$, as well as the Taylor Reynolds number $\operatorname{Re}_{\lambda}=u_{0} \lambda / \nu$, are kept constant throughout the study: $\eta / d_{b}=0.098, \lambda / d_{b}=1.0, L / d_{b}=2.1$, and $\operatorname{Re}_{\lambda}=30$. The rather low value of $\operatorname{Re}_{\lambda}$, which results in a weak separation of space and time scales, is due to the fact that the spectral methods classically used for the simulation of turbulent flows are not suitable in the presence of different fluids and that calculations performed in the physical space are much more computationally demanding (in particular because of the pressure calculation). It can be noticed that highly relevant results for the interaction between turbulence and solid particles have been obtained for similar values of $\operatorname{Re}_{\lambda}[16,17]$, and it will be shown in Sec. III that the standard features of turbulence are recovered in our simulations.

Other dimensionless parameters, independent of the turbulence level, characterize the bubble rise in a liquid at rest. In this configuration, the terminal bubble velocity and shape depend on two dimensionless groups that measure the relative strengths of the buoyancy, viscous, and surface tension forces acting on the bubble: the Bond and the Archimedes numbers. The Bond number (also known as the Eötvös number) is defined as Bo $=g d_{b}^{2} \Delta \rho / \gamma$, where $\Delta \rho \equiv \rho_{l}-\rho_{g}$ is the density difference between the liquid and the gas phases and $\gamma$ denotes the surface tension. It is set to a fixed value $\mathrm{Bo}=0.38$, which yields in quiescent conditions a nearly spherical (though deformable) bubble. This choice allows the bubble to deform without breaking up in the presence of an intense background turbulent flow. The Archimedes number (also known as the Galileo number) $\mathrm{Ar}=\sqrt{g d_{b}^{3} \rho_{l} \Delta \rho} / v$ is the variable parameter (its values are given in Table I) that determines $V_{T}$, the terminal velocity of the bubble in still liquid. This velocity is estimated herein using the correlation 
TABLE I. Bubble parameters calculated a priori in the three runs: Ar, Archimedes number; $\operatorname{Re}_{T}=d_{b} V_{T} / v$, terminal bubble Reynolds number based on $V_{T}$, the terminal velocity of the bubble in quiescent conditions; and $\beta=u_{0} / V_{T}$, turbulence intensity.

\begin{tabular}{llc}
\hline \hline $\operatorname{Ar}$ & $\operatorname{Re}_{T}$ & $\beta$ \\
\hline 40.7 & 62.5 & 0.46 \\
27.2 & 31.4 & 0.90 \\
19.2 & 17.6 & 1.60 \\
\hline \hline
\end{tabular}

of [26], which allows us to define a priori a characteristic bubble Reynolds number $\operatorname{Re}_{T}=V_{T} d_{b} / \nu$. For the range of parameters considered here, $\operatorname{Re}_{T}$ was found to be of order 10 , so in quiescent liquid the bubble motion is steady, vertical, and its wake is laminar, steady, and attached to the bubble.

\section{B. Numerical method}

Direct numerical simulations of a large deformable bubble rising in an otherwise homogeneous and isotropic turbulent flow have been performed. For this, the fluid motion must be solved in both the liquid and the gas with the appropriate jump conditions at the fluid-fluid boundary, namely, the continuity of velocity and of shear stress across the interface (owing to the absence of phase change and surface tension gradients, respectively), and a jump in normal stress equal to the surface tension force per unit area. These sets of equations coupled through interfacial jump conditions were integrated numerically using our three-dimensional DNS code, a brief description of which is given hereinafter.

In short, the incompressible Navier-Stokes equations for the two fluids are combined into a one-fluid formulation that accounts for the interface conditions and are solved by a projection method [27], the deformable gas-liquid interface is captured by a modified level-set method [28,29], and surface tension is accounted for using the continuum surface force model [30]. These are described in the following.

The velocity and pressure field are solutions of the system of equations

$$
\begin{gathered}
\frac{D(\rho \mathbf{u})}{D t}=-\nabla p+\nabla \cdot\left[\mu\left(\nabla \mathbf{u}+\nabla \mathbf{u}^{T}\right)\right]+(\rho-\langle\rho\rangle) \mathbf{g}-\gamma \boldsymbol{\kappa}+\rho \mathbf{f} H_{\varepsilon}(\psi), \\
\nabla \cdot \mathbf{u}=0,
\end{gathered}
$$

where $D / D t$ is the material time derivative, $\mathbf{u}$ stands for the velocity field, $p$ stands for the pressure one, $\mathbf{g}=-g \mathbf{e}_{z}$ is the gravitational acceleration, $\rho$ and $\mu$ respectively denote the local density and dynamic viscosity, $\langle\rho\rangle$ is the system average density that must be subtracted from the local one in the buoyancy term to prevent the entire system from accelerating in the downward vertical direction, $\gamma \kappa$ stands for the effect of surface tension (this term is nonzero at the gas-liquid interface only), and $\rho \mathbf{f}$ is a forcing term allowing us to maintain a statistically stationary level of turbulence. More details on the latter will be given in Sec. II C. The $H_{\varepsilon}(\psi)$ factor allows us to apply this term in the liquid phase only, $H_{\varepsilon}$ denoting the smoothed Heaviside function

$$
H_{\varepsilon}(\psi)= \begin{cases}1 & \text { for } \psi>\varepsilon \\ 0 & \text { for } \psi<-\varepsilon \\ \frac{1}{2}\left[1+\frac{\psi}{\varepsilon}+\frac{1}{\pi} \sin \left(\frac{\pi \psi}{\varepsilon}\right)\right] & \text { for }|\psi| \leqslant \varepsilon\end{cases}
$$

where $\varepsilon=1.5 \Delta x$ (with $\Delta x$ the grid spacing) and $\psi$ denotes the level-set function, positive in the liquid and negative in the gas. The surface tension term is calculated from the level-set function [28]

$$
\kappa=\left[\nabla \cdot\left(\frac{\nabla \psi}{|\nabla \psi|}\right)\right] \nabla H_{\varepsilon}
$$


and the (local) density and viscosity are given by

$$
\rho=H_{\varepsilon}(\psi) \rho_{l}+\left[1-H_{\varepsilon}(\psi)\right] \rho_{g}, \quad \mu=H_{\varepsilon}(\psi) \mu_{l}+\left[1-H_{\varepsilon}(\psi)\right] \mu_{g} .
$$

In our simulations, the gas-to-liquid density and viscosity ratios were set to $\rho_{g} / \rho_{l}=10^{-3}$ and $\mu_{g} / \mu_{l}=10^{-2}$, respectively. These are representative of most bubbly flows of practical interest, such as air bubbles in water.

The level-set function $\psi$ is initially defined as the signed distance to the interface and evolves by integrating an advection equation. A drawback of basic level-set methods is their poor ability to conserve the mass of each phase. Specific methods must therefore be used to solve this issue. To this aim, a source term is embedded in our code in the advection equation of the level-set function, as proposed by [29]

$$
\frac{D \psi}{D t}=A(\mathbf{u}, \psi) \psi \quad \text { with } A(\mathbf{u}, \psi)=\nabla_{i} \psi \nabla_{i} u_{j} \nabla_{j} \psi
$$

$[A(\mathbf{u}, \psi)$ is the local zeroth-order approximation of the source term in the region close to the interface], and $\psi$ is reinitialized at each time step using the procedure devised by [31]. These two improvements allow us to maintain the level-set function as a signed distance function close to the interface and thereby to improve the mass conservation. An exact mass conservation is enforced at each time step using the correction proposed by [32], which consists in slightly shifting the level-set function $\psi$ by an amount $\Delta \psi$ in such a way that the volume of each phase remains constant. We have checked that the magnitude of this correction is negligible $\left(\max |\Delta \psi| / \Delta x \lesssim 10^{-6}\right.$ in the present simulations). A more detailed description of the numerical method and the results of benchmark tests in laminar flows can be found in [33].

The governing equations (1) and (2) are solved by a projection method [27]. Our time integration algorithm is based on a third-order TVD (total-variation-diminishing) Runge-Kutta scheme for the level-set equation and on a mixed Crank-Nicolson-third-order Adams-Bashforth scheme for the Navier-Stokes equations. For spatial derivatives, we employ a standard mixed finite-differencefinite-volume discretization on a uniform Cartesian staggered grid: Fifth-order WENO (weighted essentially nonoscillatory) schemes are used for advection terms and second-order centered schemes are used otherwise. In our simulations the grid spacing was set to $\Delta x=0.64 \eta=d_{b} / 16$, a resolution found to be sufficient at the Reynolds number considered, and the Courant number based on the instantaneous maximal velocity was always less than 0.1. A more detailed description of the code as well as the results of some benchmark tests is provided in [33].

Periodic boundary conditions are applied at the boundaries of the cubic computational domain, of linear dimension $h=12 d_{b}$. This configuration effectively corresponds to a cubic array of bubbles with volume fraction of $0.03 \%$. It was shown in [33] that even at very low volume fraction a bubble rising in quiescent liquid may be affected by the wakes of its preceding neighbors. The situation is however very different here. The carrier phase is now turbulent with velocity fluctuations $u_{0}$ comparable to the bubble velocity $V_{T}\left(\beta=u_{0} / V_{T} \sim 1\right.$; see Table I). Prior work on spherical bubbles and particles set fixed in a weakly turbulent environment showed that the velocity defect in the (laminar) wake first decays as $z^{-1}$ ( $z$ being the downstream distance to the particulate) and then follows a $z^{-2}$ power law from the point where the magnitude of the velocity defect and that of the turbulent velocity fluctuations become of the same order [21,34,35]. Assuming a $z^{-2}$ decay law, a coarse estimate of the wake velocity $u_{z}$ at a downstream distance $z=h$ from the bubble is $u_{z} / u_{0} \sim\left(V_{T} / u_{0}\right)\left(h / d_{b}\right)^{-2} \sim 10^{-2} \ll 1$. It therefore seems reasonable to consider that a bubble is not affected by the wakes of its periodic images. The wake can also be characterized by the measurement of vorticity. We have checked numerically that the enstrophy statistics were not influenced by the presence of the bubble already at a distance of $2 d_{b}$ from it. The negligible effect of periodicity will also be confirmed a posteriori in Sec. III C. 


\section{Turbulence forcing}

The turbulence level was kept statistically stationary in our system through the use of a slightly modified version of the linear forcing proposed by [36], according to which the term $\mathbf{f}$ in Eq. (1) should be proportional to the velocity vector. This forcing scheme provides the advantage that it is formulated in physical space. It has been shown to yield the same results as spectral implementations of low-wave-number forcing for single-phase flow turbulence [37] and has been used in prior studies of turbulent two-phase flows in [16] (fixed solid sphere) and [38] (interface-resolved gas-liquid flow). Gravity, however, was not included in these studies. The use of the linear forcing in two-phase systems where gravity is accounted for leads to an unbounded growth of the kinetic energy and, as a consequence, does not allow a stable stationary state to be reached. However, this problem can be easily overcome by suitably modifying the expression of $\mathbf{f}$, as we now demonstrate.

In Eq. (1) the forcing $\mathbf{f}$ is defined by $\mathbf{f}=Q \mathbf{u}^{*}$, with $Q$ a positive constant. If one sets $\mathbf{u}^{*}=\mathbf{u}$ as in nonbuoyant single-phase flows [36,37], the net force on the liquid $\mathbf{N}=\rho_{l} Q \int_{\mathcal{V}_{l}} \mathbf{u}^{*} d \mathbf{x}$ is not zero, because the volume integral of $\mathbf{u}$ over the liquid phase is not strictly zero (the upward motion of the bubble must be compensated by a downflow of liquid). As a consequence, the presence of the forcing induces an exponential growth of the liquid mean flow, as observed by [19]. We solved this issue by subtracting the instantaneous mean liquid velocity $\langle\mathbf{u}\rangle_{l}$ from the local one in the definition of $\mathbf{u}^{*}$ :

$$
\mathbf{u}^{*}=\mathbf{u}-\langle\mathbf{u}\rangle_{l} \quad \text { with }\langle\mathbf{u}\rangle_{l}=\frac{1}{V_{l}} \int_{\mathcal{V}_{l}} \mathbf{u} d \mathbf{x},
$$

where $V_{l}$ is the volume of the liquid phase and $\mathcal{V}_{l}$ denotes the set of points belonging to it. With this definition, $\mathbf{N}=\mathbf{0}$ is satisfied; therefore, the forcing has no net effect on the liquid phase and a statistically stationary state can be reached. Note that even in the absence of gravity it is generally desirable to subtract the residual mean flow to ensure stability, as noticed in [16].

\section{Simulation procedure}

We now describe the simulation procedure. A carrier turbulent flow with a Taylor-microscale Reynolds number $\operatorname{Re}_{\lambda}=30$ was first generated in the periodic computational domain. An initially spherical bubble with $d_{b}=\lambda$ was then introduced and the resulting two-phase flow was evolved until a statistically stationary state was reached (this was checked by monitoring the time signals of the bubble velocity and liquid kinetic energy). The simulation was then continued over a time period of $O\left(400 T_{L}\right)$, with $T_{L}=u_{0}^{2} / \varepsilon_{0}$ the large-eddy turnover time, during which liquid Eulerian and bubble Lagrangian quantities were time averaged to get the statistics presented in Sec. III. This procedure was used for each of the values of the turbulence intensity $\beta$ considered.

As the center of mass of the bubble is not tracked explicitly in our method, computing the bubble velocity in a Lagrangian manner would have been cumbersome. The instantaneous bubble velocity V was instead calculated using the following expression:

$$
\mathbf{V}=\langle\mathbf{u}\rangle_{g} \quad \text { where }\langle\mathbf{u}\rangle_{g}=\frac{1}{V_{g}} \int_{\mathcal{V}_{g}} \mathbf{u} d \mathbf{x},
$$

where $V_{g}$ is the volume of the gas phase (that is, the bubble volume) and $\mathcal{V}_{g}$ denotes the set of points belonging to it. It has been checked that this definition yields the same result as the computation of $d \mathbf{X} / d t$, where $\mathbf{X}$ is the position of the bubble center of mass. Moreover, since the computational domain is sufficiently larger than the bubble, the velocity defined by Eq. (8) is indistinguishable from the drift velocity classically used in bubbly flows, $\mathbf{U}=\langle\mathbf{u}\rangle_{g}-\langle\mathbf{u}\rangle$, where \langle\rangle denotes a volume average over the entire domain (in our simulations, $\left|\left\langle u_{i}\right\rangle /\left\langle u_{i}\right\rangle_{g}\right| \leqslant 5 \times 10^{-4}$ for any $i \in\{x, y, z\}$, at any time and for any $\beta$ ). Such a distinction would be relevant for much larger gas volume fractions only.

For comparison purposes, we also simulated the rise of the same bubbles in otherwise quiescent liquid (using the same code with the same parameters, but with the forcing term set to zero). For each 
(a)

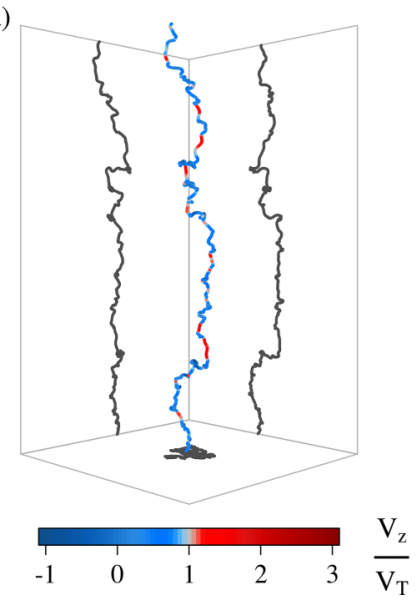

(b)

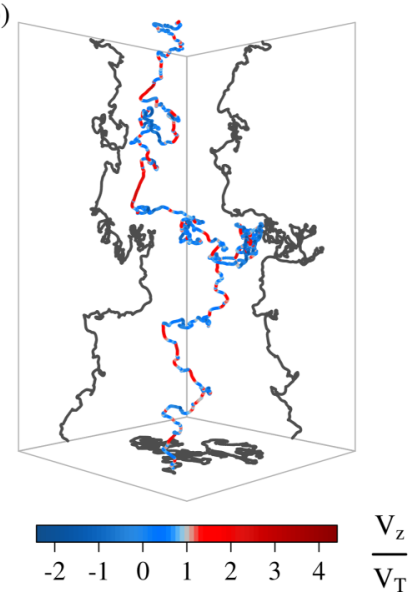

(c)

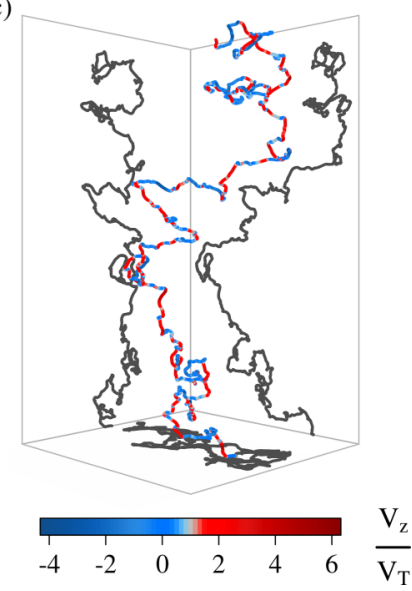

FIG. 1. Sample bubble trajectories and their projections on horizontal and vertical planes for (a) $\beta=0.46$, (b) $\beta=0.90$, and (c) $\beta=1.60$. Bubbles are rising upward; the outer box shown in light gray is a parallelepiped of width $7 h$ and height $14 h$, with $h$ the size of the computational domain. The color code shows instantaneous bubble vertical velocity normalized by the terminal velocity of the same bubble rising in still liquid.

of the three flow regimes listed in Table I we proceeded as follows. The liquid was initially at rest and the bubble, initially at rest and spherical, was allowed to rise freely through the domain under the effect of gravity. In all cases the bubble motion was perfectly rectilinear. After a transient period, the bubble velocity and shape became steady (the numerically obtained bubble velocity was constant and comparable to that predicted by the correlation of [26] used to defined $\mathrm{Re}_{T}$ in Table I and the bubble shape was nearly spherical). The bubble and liquid flow properties in quiescent conditions mentioned in Sec. III were computed at this steady state.

\section{RESULTS}

\section{A. Statistical description of the bubble motion and deformation}

In a quiescent liquid $(\beta=0)$, the bubbles considered here would rise along straight vertical paths. As the turbulence intensity $\beta$ is increased, their trajectories become more erratic and progressively deviate from vertical lines, as illustrated in Fig. 1. This section is devoted to the characterization of the bubble motion and deformation. In what follows, $\mathbf{V}$ denotes the bubble velocity, defined in Eq. (8), and $\mathbf{A}=d \mathbf{V} / d t$ is its acceleration. The overbars indicate time averaging. Subscripts $x, y$ and $z$ denote the two horizontal and the vertical components of vectors, respectively (buoyancy acts in the positive $z$ direction since $\mathbf{g}=-g \mathbf{e}_{z}$ ). Since the two horizontal directions are equivalent, Lagrangian statistics for the $x$ and $y$ components have been combined.

\section{Deformation}

We first examine the bubble deformation statistics and compare them with their values in a liquid at rest. Deformation is characterized here through the bubble sphericity, defined as the ratio between the surface of a volume-equivalent sphere and that of the bubble (lower sphericity corresponds to greater departure from a spherical shape). A spectral analysis of the sphericity time signals (not shown) reveals the absence of any dominant frequency: The bubbles do not experience periodic shape oscillations. The mean sphericity and the root mean square of its fluctuations are provided in Table II for each $\beta$. For an easier interpretation we also provide for each case the aspect ratio of a spheroid with the same sphericity. As expected, the bubble is more deformed at increasing turbulence intensity and this deformation is on average stronger in turbulent conditions than in still 
TABLE II. Bubble deformation in quiescent (subscript $T$ ) and turbulent conditions (in that case overbars indicate time averaging and the numbers in parentheses correspond to the root-mean-square fluctuations around this mean value): $\Psi$, bubble sphericity, defined as the ratio between the surface of a volume-equivalent sphere and that of the bubble, and $\chi^{\text {eq }}$, aspect ratio of an oblate spheroid with sphericity $\Psi$.

\begin{tabular}{lcccc}
\hline \hline$\beta$ & $\Psi_{T}$ & $\bar{\Psi}$ & $\chi_{T}^{\text {eq }}$ & $\overline{\chi^{\mathrm{eq}}}$ \\
\hline 0.46 & 0.9944 & $0.9918( \pm 0.0031)$ & 1.19 & $1.23(-0.05,+0.05)$ \\
0.90 & 0.9946 & $0.9919( \pm 0.0040)$ & 1.19 & $1.23(-0.07,+0.06)$ \\
1.60 & 0.9948 & $0.9869( \pm 0.0094)$ & 1.18 & $1.31(-0.16,+0.11)$ \\
\hline \hline
\end{tabular}

liquid. Due to the low value of the Bond number, it nevertheless remains overall modest, the aspect ratios of the equivalent spheroids always being smaller than 1.4.

\section{Velocity}

We now examine the statistics of the bubble velocity. Its componentwise variance and the average of $V_{z}$ are listed in Table III. The most noticeable feature here is the fact that $\overline{V_{z}}$ is significantly lower (by 60\%-77\%) than that of the same bubble in an infinite quiescent liquid. Such a reduction of the rise velocity under the effect of turbulence was already reported for much smaller bubbles $[3,5,6,39-41]$. In these investigations, a similar reduction in rise velocity was obtained for comparable $\beta$. Interestingly, the velocity reduction in our simulations is maximum when $\beta \approx 1$ (for point bubbles, both nonmonotonic [3] and monotonic [5,6] evolutions of $\overline{V_{z}} / V_{T}$ with $\beta$ have been reported, thereby indicating that the value of $\beta$ alone is not sufficient to predict the rise velocity reduction). We find that the variances of the vertical and horizontal components of $\mathbf{V}$ are of the same order. They are, up to statistical uncertainty, equal to $u_{0}^{2}$ (the variance of the liquid velocity components in a one-phase flow) for the lowest value of $\beta$ and lower than it for $\beta \gtrsim 1$.

More detailed information is provided in Fig. 2, in which the probability density functions (PDFs) of the horizontal and vertical velocity fluctuations $v_{i}=V_{i}-\overline{V_{i}}$ normalized to unit variance are plotted. These distributions are roughly Gaussian, as is widely known for tracers and small inertial particles [42], as well as for finite-size solid particles [9,17,19]. For inertial particulates, a weak asymmetry (either positive or negative) in the distribution of the vertical velocity fluctuations has been reported for point bubbles [3,6], for finite-size bubbles (although smaller than in the present simulations) [15,40], and more recently for large buoyant solid particles [19]. These prior results indicate that the shape of the vertical velocity PDF depends in a complex manner on $\beta$ and presumably on other factors such as $\mathrm{Re}_{\lambda}$ or the bubble size compared to the turbulence length scales. Since we chose to simulate the dynamics of isolated bubbles, only one object could be put in the domain and obtaining accurate high-order Lagrangian statistics is definitely out of reach. It is therefore difficult to make a definitive statement about the effect of $\beta$ on such an asymmetry

TABLE III. Nonzero mean and variance of the bubble velocity components, for each value of the turbulence intensity $\beta: V_{T}$, terminal bubble velocity in quiescent conditions, estimated from [26]; $u_{0}^{2}$, variance of the liquid velocity in the absence of the bubble; $\mathbf{V}$, bubble velocity; and $\mathbf{v}=\mathbf{V}-\overline{\mathbf{V}}$, bubble velocity fluctuation.

\begin{tabular}{lccc}
\hline \hline$\beta$ & $\overline{V_{z}} / V_{T}$ & $\overline{v_{x, y}^{2}} / u_{0}^{2}$ & $\overline{v_{z}^{2}} / u_{0}^{2}$ \\
\hline 0.46 & $0.40 \pm 0.04$ & $0.97 \pm 0.03$ & $0.98 \pm 0.05$ \\
0.90 & $0.23 \pm 0.12$ & $0.73 \pm 0.02$ & $0.77 \pm 0.04$ \\
1.60 & $0.37 \pm 0.04$ & $0.76 \pm 0.02$ & $0.70 \pm 0.04$ \\
\hline \hline
\end{tabular}



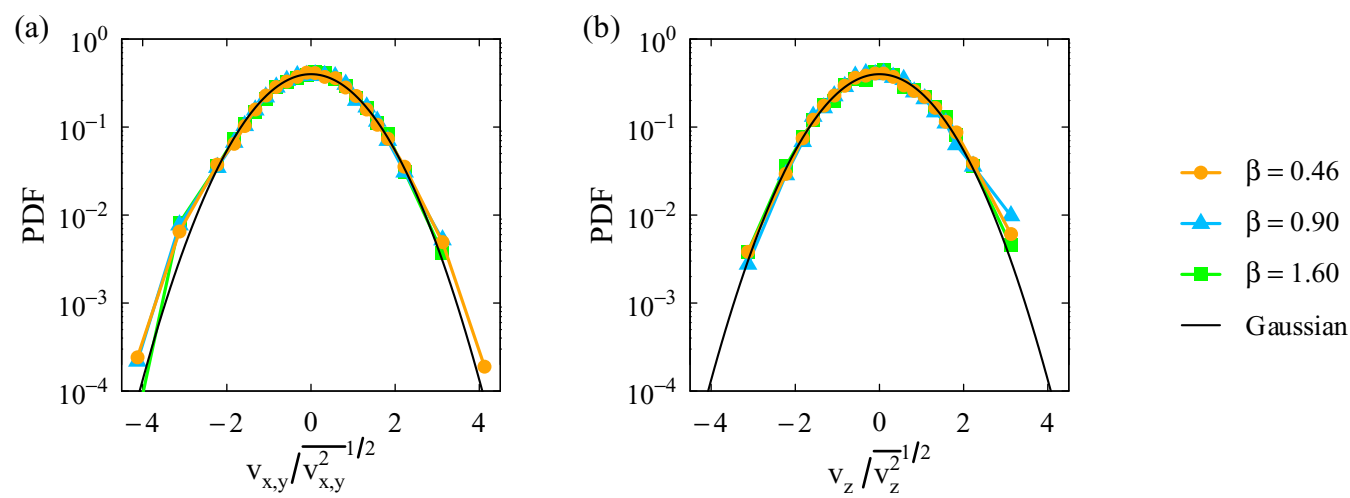

FIG. 2. PDFs of the (a) horizontal and (b) vertical components of the bubble velocity fluctuations $v_{i}=V_{i}-\overline{V_{i}}$, normalized to unit variance. The solid black line shows the Gaussian distribution.

in the distribution of the vertical velocity fluctuations. In any case, the degree of departure from Gaussianity in our simulations, if any, is small: We measured skewnesses $\left|\bar{v}_{z}^{3} / \bar{v}_{z}^{2}{ }^{3 / 2}\right|<0.3$ and flatnesses $\overline{v_{i}^{4}} /{\overline{v_{i}^{2}}}^{2}=3.1 \pm 0.3$ for $i=x, y, z$. Finally, although direct comparison to prior work on small bubbles is not possible (essentially because of the mismatch in the values of $\operatorname{Re}_{\lambda}, d_{b} / L$, and $\operatorname{Re}_{T}$ ), it is worth mentioning that nearly Gaussian vertical velocity distributions have also been obtained for $\beta \approx 0.5[3,40]$ and for $\beta \approx 1.2$ [6].

\section{Acceleration}

We now turn to the statistics of the bubble acceleration components. The mean bubble acceleration is zero and the componentwise acceleration variance normalized by $g^{2}$ is reported in Table IV. For any $\beta$ the horizontal and vertical variances are of the same order. They depend on $\beta: \overline{a_{i}^{2}} \approx g^{2}$ for the smallest value of $\beta$ considered here, when buoyancy effects are the strongest, and $\overline{a_{i}^{2}} \approx 10 g^{2}$ for the largest $\beta$.

Figure 3 represents the PDFs of the horizontal and vertical components of the bubble acceleration fluctuations $a_{i}=A_{i}-\overline{A_{i}}$ normalized to unit variance. These PDFs are highly non-Gaussian and exhibit large tails, as already evidenced for fluid tracers and small inertial particles [42] and more recently for finite-size solid particles $[9,17,19,43]$. As shown by the dashed line in Fig. 3(a), our data for the horizontal components of acceleration are well fitted by a log-normal distribution

$$
p\left(a_{i}\right)=\frac{e^{3 \sigma^{2} / 2}}{4 \sqrt{3}}\left[1-\operatorname{erf}\left(\frac{\ln \left(\left|a_{i} / \sqrt{3}\right|\right)+2 \sigma^{2}}{\sqrt{2} \sigma}\right)\right],
$$

TABLE IV. Variance of the bubble acceleration components for each value of the turbulence intensity $\beta: \mathbf{a}=\mathbf{A}-\overline{\mathbf{A}}$, bubble acceleration fluctuation, and $g$, magnitude of the gravitational acceleration.

\begin{tabular}{lcc}
\hline \hline$\beta$ & \multicolumn{1}{c}{$\overline{a_{x, y}^{2}} / g^{2}$} & $\overline{a_{z}^{2}} / g^{2}$ \\
\hline 0.46 & $1.23 \pm 0.01$ & $1.08 \pm 0.01$ \\
0.90 & $3.03 \pm 0.03$ & $3.12 \pm 0.06$ \\
1.60 & $10.14 \pm 0.40$ & $9.51 \pm 0.80$ \\
\hline \hline
\end{tabular}



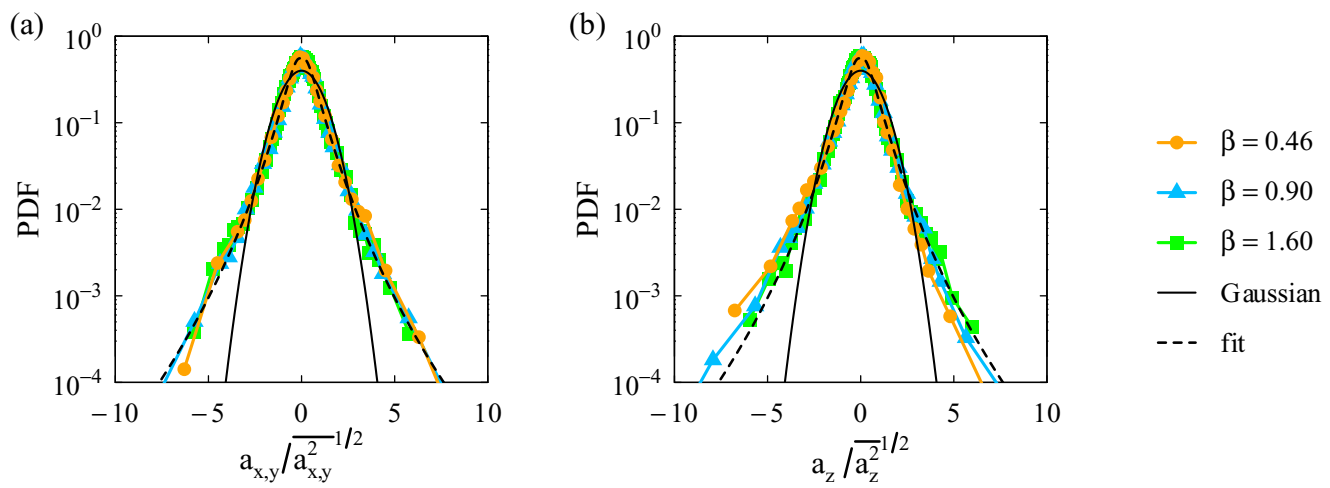

FIG. 3. PDFs of the (a) horizontal and (b) vertical components of the bubble acceleration fluctuation $a_{i}=A_{i}-\overline{A_{i}}$, normalized to unit variance. The solid black line shows the Gaussian distribution and the dashed black line the log-normal fit of the horizontal component distributions [Eq. (9) with $\sigma=0.66$ ].

proposed in [44] for tracers. The best fit (in the sense of least squares) is obtained for $\sigma=0.66 \pm 0.01$, which corresponds to a distribution flatness $\overline{a_{i}^{4}} /{\overline{a_{i}^{2}}}^{2}=10.3$. As a comparison, the same relation was found to describe correctly experimentally obtained acceleration statistics of rigid particles of different sizes and densities, with a flatness of 8.5 [43,45]. Other data numerically obtained for finite-size neutrally buoyant solid particles exhibited the same behavior, with flatnesses between 6 and $8.5[17,19]$. The previously mentioned fits would be nearly indistinguishable from ours and are therefore not shown in Fig. 3(a). For buoyant solid particles with $d_{b}=6.7 \eta=0.42 \lambda$, Ref. [19] obtained a noticeably smaller flatness of 3.9 for the horizontal component of the particle acceleration, although no explanation could be offered for this low value (on a side note, it is not clear that their particles can be strictly considered as isolated).

An additional property evidenced by our simulations is the negative asymmetry of the distribution of the vertical acceleration component, particularly visible for the most buoyant bubble $(\beta=0.46)$. In quantitative terms, we measured skewnesses ${\overline{a_{z}^{3}}}_{/ \bar{a}^{2}}^{3 / 2}=\{-1.0 \pm 0.3,-0.6 \pm 0.6,-0.5 \pm 0.2\}$ for $\beta=\{0.46,0.90,1.60\}$, although some reservations must be expressed regarding the accuracy of these values given the limited amount of data from which they are computed. Since the vertical direction is parallel to the average direction of motion, this feature may be reminiscent of the negative skewness of the longitudinal acceleration distribution, a property recently evidenced for fluid tracers $[46,47]$.

To support this hypothesis, we show in Fig. 4 the PDF of the longitudinal acceleration $A_{\|}=$ $\mathbf{A} \cdot \mathbf{V} /|\mathbf{V}|$. The mean of $A_{\|}$is zero, but its distribution is undoubtedly negatively skewed for all the considered values of $\beta$ : Its skewness is $\overline{A_{\|}^{3}} /{\overline{A_{\|}^{2}}}^{3 / 2}=-0.6 \pm 0.1$ (with the same reservations about accuracy as above) and is virtually independent of $\beta$. Therefore, the $\beta$ dependence of the vertical acceleration distribution in Fig. 3(b) can be explained by the fact that the bubble velocity is more and more preferentially oriented in the vertical direction, that is, the bubble path gets closer to a vertical line, at decreasing $\beta$ (see Fig. 1). Furthermore, the distribution of the longitudinal acceleration of an inertial object is shown to be negatively skewed. Since the longitudinal acceleration represents the velocity magnitude rate of change $\left(A_{\|}=d|\mathbf{V}| / d t\right)$, this means that a finite-size bubble undergoes, on average, stronger deceleration than (positive) acceleration. We have checked that this holds as well in a frame moving at the bubble velocity: The PDF of $a_{\|}=\mathbf{a} \cdot \mathbf{v} /|\mathbf{v}|$ is similar to that of $A_{\|}$, with a negative skewness of the same order. In the Lagrangian framework, the negative asymmetry of the longitudinal acceleration distribution of tracers is a signature of the time irreversibility of turbulence. Finally, it is worthwhile to note that the same analysis could provide an interpretation of 


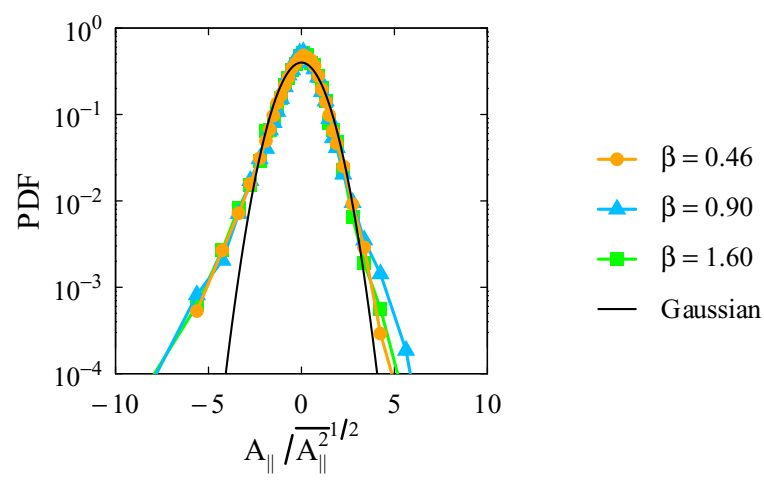

FIG. 4. PDF of the bubble longitudinal acceleration $A_{\|}=\mathbf{A} \cdot \mathbf{V} /|\mathbf{V}|$, normalized to unit variance. The solid black line shows the Gaussian distribution.

the positively skewed shape (skewness of 0.63) of the vertical acceleration PDF recently reported for finite-size sedimenting solid particles [19].

\section{B. Modeling of hydrodynamic forces}

In the present simulations, the bubbles remain close to spherical, as shown in Table II. An often-used equation of motion for a spherical bubble at moderately high Reynolds number in an arbitrary flow is [48]

$$
\begin{aligned}
\mathbf{A}= & \frac{\alpha-1}{\alpha+C_{M}} \mathbf{g}+\frac{1+C_{M}}{\alpha+C_{M}} \frac{D \mathbf{U}_{0}}{D t}+\frac{C_{L}}{\alpha+C_{M}}\left(\mathbf{U}_{0}-\mathbf{V}\right) \times \mathbf{\Omega}_{0}+\frac{1}{\alpha+C_{M}} \frac{3 C_{D}}{4 d_{b}}\left|\mathbf{U}_{0}-\mathbf{V}\right|\left(\mathbf{U}_{0}-\mathbf{V}\right) \\
& +\frac{1}{\alpha+C_{M}} \frac{18 v}{d_{b}^{2}} \int_{-\infty}^{t} K(t-\tau) \frac{d\left(\mathbf{U}_{0}-\mathbf{V}\right)}{d \tau} d \tau
\end{aligned}
$$

where $\mathbf{A}=d \mathbf{V} / d t$ is the bubble acceleration, $\alpha=\rho_{g} / \rho_{l}$ denotes the gas-to-liquid density ratio, and $\mathbf{U}_{0}$ and $\boldsymbol{\Omega}_{0}$ are, respectively, the undisturbed fluid velocity and vorticity at the bubble position. This approximate equation derives from a force balance that includes buoyancy (yielding the first term on the right-hand side), undisturbed liquid acceleration and acceleration reaction force (resulting, when combined, in the second term), lift (third term), steady drag (fourth term), and history force (last term). In addition, $C_{M}, C_{L}$, and $C_{D}$ are the corresponding added mass, lift, and steady drag coefficients, respectively, and $K$ is the history kernel. Equation (10) assumes length and time scales of the flow to be large compared to those of the bubble. The validity of adding these various forces is not clear a priori and some of the coefficients in it may depend on a Reynolds number and a shear rate, as discussed further in [48].

Despite these reservations, Eq. (10) may provide a first approximation of the bubble dynamics. A significant further issue with it lies in the fact that the notion of unperturbed flow at bubble position is then irrelevant, since the base flow varies over length scales smaller than $O\left(d_{b}\right)$ and the bubble modifies it in its neighborhood. A first step toward the extension of Eq. (10) to finite-size bubbles therefore consists in finding adequate definitions for $\mathbf{U}_{0}$ and $\boldsymbol{\Omega}_{0}$. A conceivable approach, first proposed in [20], consists in defining these quantities as volume averages of the fluid velocity and vorticity over appropriate volumes in the bubble surroundings. This idea was proven successful in [16], which showed that the fluid angular velocity appearing in the torque equation of a fixed solid particle much larger than $\eta$ can be defined in terms of the fluid vorticity averaged over a shell concentric with the object.

We undertake here an approach similar to that used in [16]: The idea is to replace $\mathbf{U}_{0}\left(\boldsymbol{\Omega}_{0}\right)$ in Eq. (10) by its counterpart $\langle\mathbf{u}\rangle_{s}\left(\langle\boldsymbol{\omega}\rangle_{s}\right.$, with $\left.\boldsymbol{\omega}=\boldsymbol{\nabla} \times \mathbf{u}\right)$ defined as the average of the local liquid 
velocity (vorticity) over a volume comprised between the gas-liquid interface and a surface located at a distance $s$ from it. Formally, this average reads

$$
\langle\mathbf{q}\rangle_{s}(t)=\frac{1}{V_{s}(t)} \int_{\mathcal{V}(s, t)} \mathbf{q}(\mathbf{x}, t) d \mathbf{x} \quad \text { with } V_{s}(t)=\int_{\mathcal{V}(s, t)} d \mathbf{x},
$$

where $\mathcal{V}(s, t)$ contains the points in the liquid phase such that $0 \leqslant \psi(\mathbf{x}, t) \leqslant s$ at time $t$, with $\psi$ the normal distance to the interface. If this volume-averaging approach is appropriate and if Eq. (10) provides a descent approximation of the bubble dynamics, it might be possible to find a value of $s$ for which the bubble acceleration $\mathbf{A}$ is reasonably correlated to $d\langle\mathbf{u}\rangle_{s} / d t,\left(\langle\mathbf{u}\rangle_{s}-\mathbf{V}\right)$, and $\left(\langle\mathbf{u}\rangle_{s}-\mathbf{V}\right) \times\langle\boldsymbol{\omega}\rangle_{s}$. Given that the adequate shell thickness $s$ may a priori depend on the nature of the force involved, we will treat the second, third, and fourth terms on the right-hand side of Eq. (10) separately. Owing to the lack of a reliable expression of the history kernel $K$ for a bubble in nonrectilinear motion at finite Reynolds number [48], the history term cannot be treated rigorously and is therefore not investigated.

We first investigate the second term on the right-hand side of Eq. (10), which represents the combination of the acceleration reaction force and the effect of the undisturbed fluid acceleration and in which the unknown variable is $\mathbf{U}_{0}$. Under the assumption of a near-spherical bubble shape, $C_{M}$ is constant and is unimportant for the present purpose since it only affects the force magnitudes, not their correlation with the acceleration. We will now determine the thickness $s_{u}^{\text {acc }}$ of the shell over which $\mathbf{u}$ should be averaged for estimating $\mathbf{U}_{0}$ at best. Recalling that part of the force arises from the undisturbed fluid acceleration integrated over the bubble volume, it seems reasonable to expect the shell volume to be comparable to it, which yields an expected value of $s_{u}^{\text {acc }}=0.13 d_{b}$, assuming the bubble to be nearly spherical. The optimal shell thickness $s_{u}^{\text {acc }}$ has been determined from our simulations by maximizing the componentwise correlation between $\mathbf{A}$ and $\mathbf{F}^{\text {acc }}$, the latter being defined by

$$
\mathbf{F}^{\mathrm{acc}}=\frac{d\langle\mathbf{u}\rangle_{s_{u}}}{d t}
$$

in which the unimportant factor $\left(1+C_{M}\right) /\left(\alpha+C_{M}\right)$ is omitted. The maximum correlation coefficients were obtained for $s_{u}^{\text {acc }} / d_{b}=0.15 \pm 0.05$ whatever $\beta$, in agreement with our expectations. They are remarkably high (between 0.85 and 0.89 , depending on $\beta$ and on the component considered), as shown in Table V. This strong correlation is also visible in Fig. 5, in which the componentwise joint PDFs of $\mathbf{A}$ and $\mathbf{F}^{\text {acc }}$ are plotted for $\beta=0.46$. The results obtained for the other values of $\beta$ are not shown but display the same behavior.

We now turn to the lift contribution to the bubble acceleration [third term on the right-hand side of Eq. (10)], which involves three unknown quantities $C_{L}, \mathbf{U}_{0}$, and $\boldsymbol{\Omega}_{0}$. The lift coefficient $C_{L}$ for a

TABLE V. Thicknesses of the shells over which the liquid velocity and vorticity should be averaged in the estimates of $\mathbf{U}_{0}$ and $\boldsymbol{\Omega}_{0}$, which maximize the componentwise correlation between the bubble acceleration $\mathbf{A}$ and hydrodynamic forces in Eq. (10), and values of the associated correlation coefficients: $s_{u}$ and $s_{\omega}$, thickness of the shells over which the liquid velocity and vorticity are respectively averaged; $C_{x, y}$ and $C_{z}$, correlation coefficients between the horizontal and vertical components of $\mathbf{A}$ and of the forces; superscripts acc and lift respectively refer to the second and third terms on the right-hand side of Eq. (10); and $\delta$, thickness of the viscous boundary layer, estimated from $\delta / d_{b}=\sqrt{2 / \operatorname{Re}_{b}}$. The uncertainty on $s_{u}^{\text {acc }} / d_{b}$ and $s_{\omega}^{\text {lift }} / d_{b}$ is \pm 0.05 and the uncertainty on $s_{u}^{\text {lift }} / d_{b}$ is \pm 0.25 .

\begin{tabular}{lcccccccc}
\hline \hline$\beta$ & $s_{u}^{\text {acc }} / d_{b}$ & $C_{x, y}^{\text {acc }}$ & $C_{z}^{\text {acc }}$ & $s_{u}^{\text {lift }} / d_{b}$ & $s_{\omega}^{\text {lift }} / d_{b}$ & $C_{x, y}^{\text {lift }}$ & $C_{z}^{\text {lift }}$ & $\delta / d_{b}$ \\
\hline 0.46 & 0.15 & 0.88 & 0.86 & 1.25 & 0.20 & 0.77 & 0.76 & $0.22(-0.04,+0.07)$ \\
0.90 & 0.15 & 0.87 & 0.87 & 1.25 & 0.25 & 0.66 & 0.68 & $0.27(-0.05,+0.11)$ \\
1.60 & 0.15 & 0.86 & 0.85 & 1.00 & 0.30 & 0.63 & 0.61 & $0.30(-0.06,+0.12)$ \\
\hline \hline
\end{tabular}



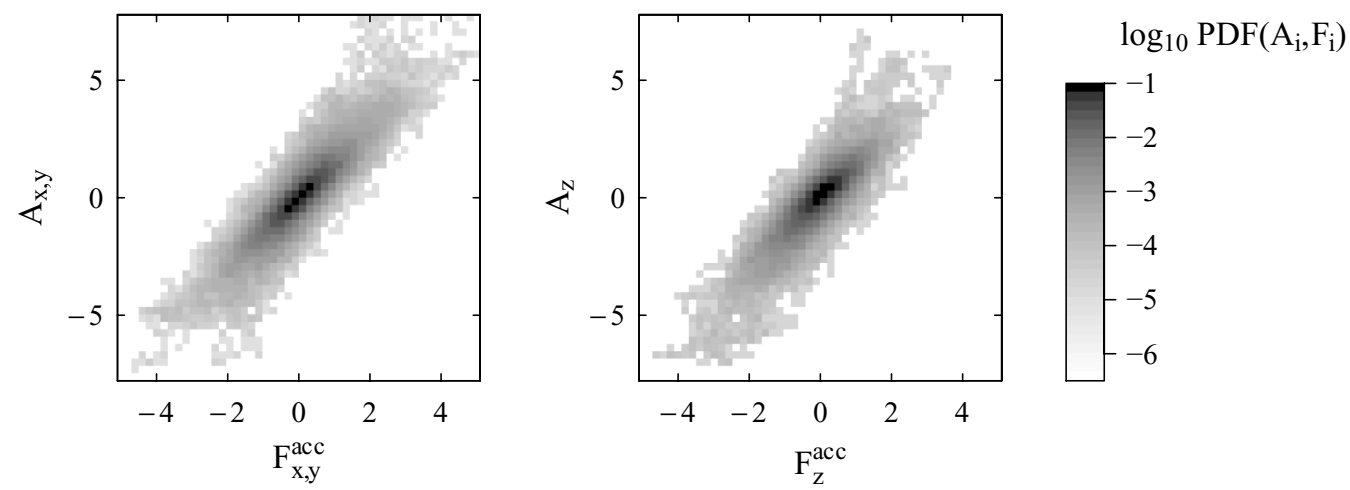

FIG. 5. Componentwise joint PDFs (logarithmic grayscale) of the bubble acceleration $\mathbf{A}$ and $\mathbf{F}^{\text {acc }}=d\langle\mathbf{u}\rangle_{s_{u}^{\text {acc }}} / d t$, for $\beta=0.46$. The value of $s_{u}^{\text {acc }}$ is given in Table V.

spherical bubble depends a priori on the Reynolds number, the shear rate, and possibly other factors $[49,50]$, but will be assumed to be constant in the results presented hereafter (we have checked that using the expression of $C_{L}$ proposed by [49], which includes dependences on Reynolds number and shear rate, yields identical results). Under this assumption, its exact value is unimportant for the present purpose. The optimal shell thicknesses $s_{u}^{\text {lift }}$ and $s_{\omega}^{\text {lift }}$ are determined by maximizing the correlation between $\mathbf{A}$ and $\mathbf{F}^{\text {lift }}$, the latter being defined by

$$
\mathbf{F}^{\text {lift }}=\left(\langle\mathbf{u}\rangle_{s_{u}^{\text {lift }}}-\mathbf{V}\right) \times\langle\boldsymbol{\omega}\rangle_{s_{\omega}^{\text {lift }}},
$$

in which the unimportant factor $C_{L} /\left(\alpha+C_{M}\right)$ is omitted. The results are summarized in Table V. The largest correlation coefficients are approximately $\{0.8,0.7,0.6\}$ for $\beta=\{0.46,0.90,1.60\}$. They were obtained by estimating $\mathbf{U}_{0}$ as the volume average of velocity over distances $O\left(d_{b}\right)$ from the interface and $\boldsymbol{\Omega}_{0}$ as the volume average of vorticity over smaller volumes of liquid, more precisely over distances $O(\delta)$, where $\delta$ is the thickness of a loosely defined "boundary layer," estimated as $\delta / d_{b} \sim \sqrt{2 / \operatorname{Re}_{b}}$ [51], where $\operatorname{Re}_{b}$ is the bubble Reynolds number defined as $\operatorname{Re}_{b}=\overline{\mid\langle\mathbf{u}\rangle_{s_{u}} \text { lift }-\mathbf{V} \mid} d_{b} / v$ (removing the time average in the definition of $\mathrm{Re}_{b}$ does not affect the results). Incidentally, the undisturbed fluid vorticity entering in the torque equation of a large solid particle in turbulence was found to be well estimated by averaging vorticity in the same volume (up to distances $\sim \delta$ from the particle surface) [16]. The reasonable correlation between $\mathbf{A}$ and $\mathbf{F}^{\text {lift }}$ when $s_{u}^{\text {lift }}$ and $s_{\omega}^{\text {lift }}$ have the values given in Table $\mathrm{V}$ is also illustrated in Fig. 6, in which typical joint PDFs between these
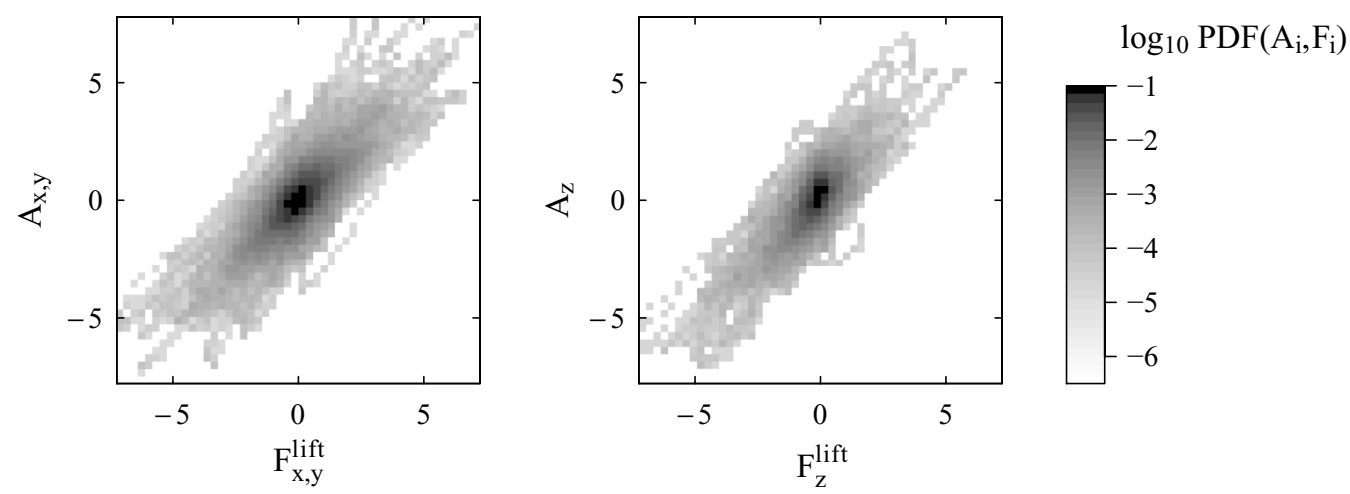

FIG. 6. Componentwise joint PDFs (logarithmic grayscale) of the bubble acceleration $\mathbf{A}$ and $\mathbf{F}^{\text {lift }}=\left(\langle\mathbf{u}\rangle_{s_{u}^{\text {lift }}}-\mathbf{V}\right) \times\langle\boldsymbol{\omega}\rangle_{s_{\omega}^{\text {lift }}}$, for $\beta=0.46$. The values of $s_{u}^{\text {lift }}$ and $s_{\omega}^{\text {lift }}$ are given in Table V. 
two quantities are shown. It is worth mentioning that $\mathbf{F}^{\text {lift }}$ is, on average, directed downward, which indicates that the force associated with $\mathbf{F}^{\text {lift }}$ acts, on average, against the bubble rise. This result is compatible with the mechanisms known to explain the turbulence-induced reduction of rise velocity of point bubbles, which will be summarized at the beginning of Sec. III C.

The same procedure is used for evaluating the liquid velocity $\mathbf{U}_{0}$ entering in the expression of the drag force [fourth term on the right-hand side of Eq. (10)]. As $C_{D}$ is expected to depend (at least) on a (properly defined) Reynolds number [48], we investigate the correlation between $\mathbf{A}$ and $\mathbf{F}^{\mathrm{drag}}$, where the latter is defined by

$$
\mathbf{F}^{\text {drag }}=C_{D}\left|\langle\mathbf{u}\rangle_{s_{u}^{\text {drag }}}-\mathbf{V}\right|\left(\langle\mathbf{u}\rangle_{s_{u}^{\text {drag }}}-\mathbf{V}\right) .
$$

For $C_{D}$ we use the empirical correlation of [26], which is valid for a spherical bubble in uniform flow at any Reynolds number (the latter being based on the slip velocity magnitude $\left|\langle\mathbf{u}\rangle_{s_{u}^{\text {drag }}}-\mathbf{V}\right|$ ). Whatever distance $s_{u}^{\mathrm{drag}}$ from the interface over which the liquid velocity is averaged, the correlation coefficient between $\mathbf{A}$ and $\mathbf{F}^{\text {drag }}$ is always found to be lower than 0.1. Using simpler expressions of $C_{D}$ or including a correction for shear [48] does not increase this correlation. A possible reason for this absence of correlation is the inadequacy of available expressions of $C_{D}$ in the case of strongly nonuniform flows. Another explanation is the inappropriateness of the shell-averaging approach for the drag term as modeled in Eq. (10). A third possibility is that the drag force might essentially balance buoyancy and thereby contribute only marginally to the bubble acceleration fluctuations. In any case, it must be stressed that Eq. (10) is not necessarily expected to hold in the present configuration.

\section{Preferential sampling}

Experiments on the motion of small bubbles $\left(d_{b} \lesssim \eta\right)$ in homogeneous isotropic turbulence demonstrated that the rise velocity of bubbles is generally reduced by turbulence [40,41], hence confirming predictions of point-bubble simulations [1-6]. This result is extended by our simulations to the regime of large bubbles, as shown in Sec. III A. Two mechanisms retarding the rise of small bubbles have been identified depending on $\beta$. For lower values of $\beta$, bubbles rise fast through the flow and are transported toward downflow regions by lift forces, where their velocity is reduced owing to the increased viscous drag and to the downward lift force induced by the bubble lateral motion [3]. For higher values of $\beta$, bubbles are trapped inside vortices [1,2,52] and preferentially accumulate on the downflow side of eddies under the effect of the lift force [3-5], which further reduces their rise velocity. According to these mechanisms, small bubbles should sample preferentially downflow and/or vortical regions, features confirmed by numerical simulations of point bubbles $[3,5,6]$. An important question to be answered is whether or not large bubbles sample the flow uniformly.

In point-bubble simulations, increased residence time in downflow regions and accumulation in vortices can be easily quantified by averaging the liquid vertical velocity and enstrophy at the bubble's position. When the bubble is much larger than the smallest length scales of the flow, two difficulties arise: First, the bubble might be larger than the regions of interest, and second, because the presence of the bubble induces local disturbances in the ambient flow, this effect cannot be directly disentangled from that of preferential sampling. The present proposal to characterize the flow sampled by the bubble consists of three steps.

(i) The typical size of the regions of interest is first compared with $d_{b}$. If the former is smaller than the latter, then the notion of residence of the bubble in these areas is meaningless.

(ii) A conditional averaging of the liquid flow in the vicinity of the bubble along its path is performed.

(iii) The resulting statistics are compared with those obtained in the case where the bubble rises steadily in still liquid.

If the results of (ii) and (iii) are sufficiently different (in magnitude or in sign) and if the characteristic size of the sampled regions is large enough, then a qualitative estimate of the sole contribution of preferential sampling can be inferred. 
(a)

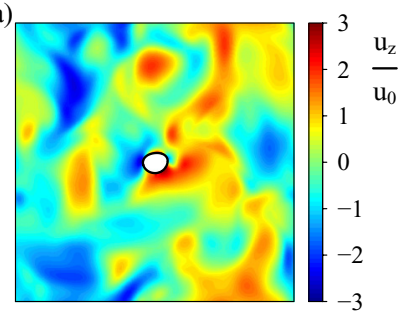

(b)

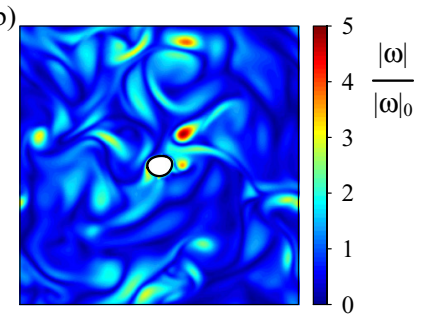

(c)

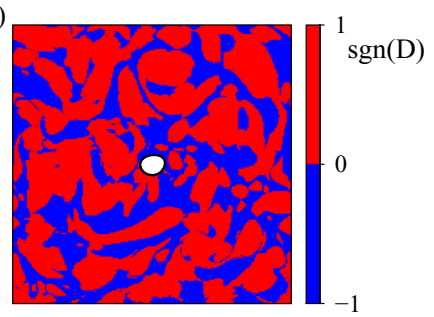

FIG. 7. Simultaneous snapshots of the carrier flow in a plane passing through the bubble center: (a) vertical component of velocity $u_{z}$, (b) vorticity magnitude $|\omega|$, and (c) sign of $D$ (defined in the main text), used to differentiate between strain-dominated $(D<0)$ and vorticity-dominated $(D>0)$ regions. Velocity and vorticity are normalized by their mean values in the absence of the bubble. For clarity the bubble interior is colored in white and the gas-liquid interface is depicted by a black line. Note that the single-phase flow obtained without the bubble has similar characteristics.

\section{Definition of the conditional averaging procedures}

Information about the local flow around the bubble is collected by a conditional averaging of the liquid flow in the vicinity of the bubble along its path. We introduce a polar coordinate system $(r, \theta)$ with its origin located at the bubble center of mass $\mathbf{X}$ and oriented along its instantaneous (absolute) direction of motion $\mathbf{V}$ : Here $r=|\mathbf{r}|$, where $\mathbf{r}=\mathbf{x}-\mathbf{X}$ is the position vector relative to the bubble center of mass, and $\theta=\arccos \left(\frac{\mathbf{r}}{|\mathbf{r}|} \cdot \frac{\mathbf{V}}{|\mathbf{V}|}\right)$ is the angle between $\mathbf{r}$ and the bubble instantaneous velocity $\mathbf{V}$. The average of any quantity $q$ of interest conditioned on $r$ and $\theta$ is calculated as follows ( $T$ is the total duration of the simulation):

$$
\langle q\rangle_{r, \theta}=\frac{1}{T} \int_{0}^{T}\left(\frac{1}{V_{r, \theta}(t)} \int_{\mathcal{V}(r, \theta, t)} q(\mathbf{x}, t) d \mathbf{x}\right) d t \quad \text { with } V_{r, \theta}(t)=\int_{\mathcal{V}(r, \theta, t)} d \mathbf{x},
$$

where $\mathcal{V}(r, \theta, t)$ is the set of points $\left(r^{\prime}, \theta^{\prime}\right)$ such that $r^{\prime} \in[r-\Delta x / 2, r+\Delta x / 2]$ (with $\Delta x$ the grid spacing) and $\theta^{\prime} \in\left[\theta-1^{\circ}, \theta+1^{\circ}\right]$ at time $t$.

We also compute the average of $q$ conditioned on the distance to the bubble surface $\psi=$ $\min \left(\mid \mathbf{x}-\mathbf{X}_{\text {interface }}\right)$, with $\mathbf{X}_{\text {interface }}$ the set of points lying on the interface. This average is defined as

$$
\langle q\rangle_{\psi}=\frac{1}{T} \int_{0}^{T}\left(\frac{1}{V_{\psi}(t)} \int_{\mathcal{V}(\psi, t)} q(\mathbf{x}, t) d \mathbf{x}\right) d t \quad \text { with } V_{\psi}(t)=\int_{\mathcal{V}(\psi, t)} d \mathbf{x},
$$

where $\mathcal{V}(\psi, t)$ is the set of points located at a distance $\psi^{\prime} \in[\psi-\Delta x / 2, \psi+\Delta x / 2]$ from the interface at time $t$.

\section{Preferential sampling of the downflow regions}

We first assess whether the bubble spends more time in downward velocity regions. As expected, the characteristic size of these regions [blue areas in Fig. 7(a)] is found to be comparable to the integral length scale $L$ and is therefore larger than $d_{b}\left(L \approx 2 d_{b}\right)$. The conditionally averaged field $\left\langle u_{z}\right\rangle_{r, \theta}$ is shown in Fig. 8 (top row) for increasing $\beta$ from left to right. The profile of $u_{z}$ as a function of the distance to the interface $\psi$ is presented in Fig. 9(a); for comparison, the results obtained without background turbulence are shown in the inset. A bubble rising in an otherwise quiescent liquid pushes the liquid aside and drags some liquid with it; this results in an average upflow in its immediate vicinity, which can be seen in the inset of Fig. 9(a). In the presence of a turbulent carrier flow, this bubble-induced upflow is still present [it is clearly visible in Fig. 8 for $\beta=0.46$, which corresponds to the most buoyant (or fastest) bubble] and is responsible for the sharp increase in $\left\langle u_{z}\right\rangle_{\psi}$ with decreasing distance very close to the interface. We will therefore ignore this effect. Farther from the interface, for $\beta=1.60,\left\langle u_{z}\right\rangle_{\psi}$ and $\left\langle u_{z}\right\rangle_{r, \theta}$ are approximately zero, meaning that 

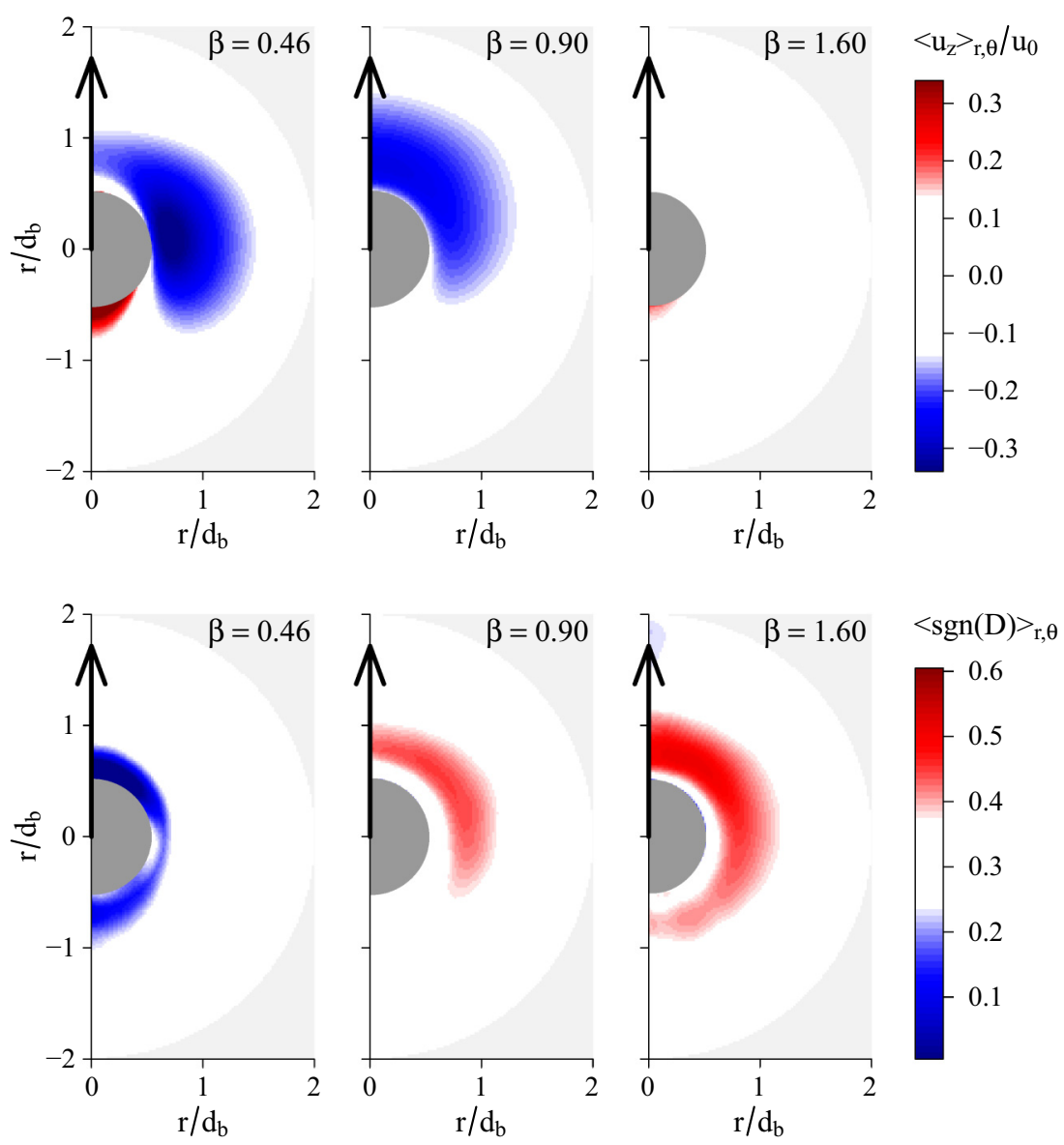

FIG. 8. Average flow field around the bubble, conditioned on $r$ and $\theta$ [see Eq. (15)] for increasing $\beta$ from left to right. The top row shows the vertical velocity, normalized by $u_{0}$. The bottom row shows the sign of the discriminant $D$ used to differentiate between strain-dominated $(D<0)$ and vorticity-dominated $(D>0)$ regions (the color scale is centered on the single-phase flow value). The arrows indicate the bubble instantaneous direction of motion.

the bubble samples equally upflow and downflow regions. In contrast, for $\beta=0.46$ and 0.90 , the vertical velocity is, on average, clearly negative around the bubble, thereby revealing a significant preference of the bubbles for downward velocity regions.

\section{Preferential sampling of the swirling regions}

As previously mentioned, small bubbles for which $\beta$ is large are known to be trapped in vortices. Since vorticity is a quantity varying over small scales [as illustrated in Fig. 7(b)], preferential sampling of vortical zones cannot be evidenced by averaging the vorticity around the bubble. Alternatively, the topology of the flow can be characterized by the eigenvalues of the velocity gradient tensor: If they are all real, the flow is locally dominated by strain, whereas if two of them are complex conjugates, the flow is locally swirling. In incompressible flows these eigenvalues only depend on $Q$ and $R$, the second and third invariants of the velocity gradient tensor, respectively $[53,54]$. It follows that the nature of the eigenvalues only depends on the sign of the discriminant $D=27 R^{2}+4 Q^{3}$ : If $D<0$ the three eigenvalues are real; if $D>0$ two of them are complex conjugates. It can be observed in Fig. 7(c), where we show an instantaneous snapshot of $\operatorname{sgn}(D)$ (the 

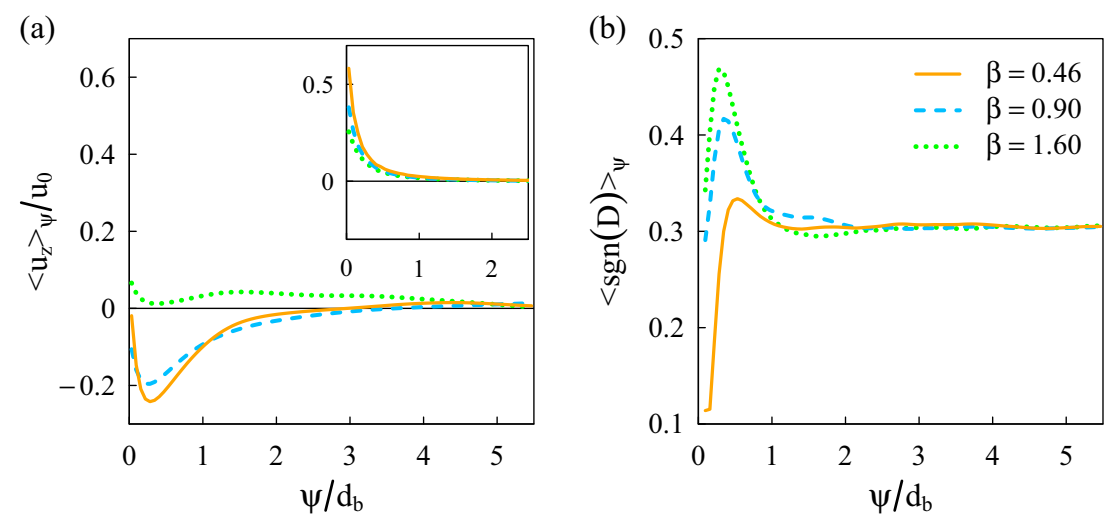

FIG. 9. Average flow profile around the bubble, conditioned on the distance to the interface [see Eq. (16)]: (a) vertical velocity normalized by $u_{0}$ (the inset shows the same quantity in the absence of turbulence) and (b) sign of the discriminant $D$ used to differentiate between strain-dominated $(D<0)$ and vorticity-dominated $(D>0)$ regions.

sign of the discriminant), that strain-dominated $(D<0)$ and vorticity-dominated $(D>0)$ regions defined in that way are of size comparable to or larger than that of the bubble. As a consequence, increased residence time of the bubble in swirling regions would be meaningful if one would define them as areas of positive $D$.

The conditionally averaged field $\langle\operatorname{sgn}(D)\rangle_{r, \theta}$ is presented in Fig. 8 (bottom row) and the evolution of $\langle\operatorname{sgn}(D)\rangle_{\psi}$ with the distance to the interface $\psi$ is shown in Fig. 9(b). Far from the bubble, $\langle\operatorname{sgn}(D)\rangle_{\psi}$ is equal to $\langle\operatorname{sgn}(D)\rangle_{0} \approx 0.3$, the mean value of $\operatorname{sgn}(D)$ in the single-phase flow [loosely speaking, $\langle\operatorname{sgn}(D)\rangle_{0}>0$ means that swirling regions occupy a larger volume than strain-dominated regions in the base flow]. As the distance to the bubble surface reduces, an increase in $\langle\operatorname{sgn}(D)\rangle_{\psi}$ is observed, followed by a sharp decrease at distances smaller than one bubble radius. Since for a single bubble rising in still liquid we obtain $-1.0 \leqslant\langle\operatorname{sgn}(D)\rangle_{\psi} \leqslant-0.8$ for all $\psi$ and since this sharp decrease occurs extremely close to the interface, we conjecture that this local reduction of $\langle\operatorname{sgn}(D)\rangle_{\psi}$ results from the distortion of the flow by the bubble. Ignoring this effect, it can be noticed that for $\beta=0.46,\langle\operatorname{sgn}(D)\rangle_{\psi}$ and $\langle\operatorname{sgn}(D)\rangle_{r, \theta}$ are not significantly modified near the bubble. This means that the time spent by the bubble in regions dominated by strain and by vorticity is roughly proportional to their respective volumes. The situation is different for $\beta=0.90$ and 1.60. A large red region around the bubble can be identified in Fig. 8 (bottom row), corresponding to $\langle\operatorname{sgn}(D)\rangle_{r, \theta}$ greater than $\langle\operatorname{sgn}(D)\rangle_{0}$. This relative increase, also clearly visible in Fig. 9(b), indicates that the flow sampled by the bubble is biased: For $\beta=0.90$ and 1.60 , the bubble preferentially resides in swirling regions $[\operatorname{sgn}(D)=1]$ of the flow. This effect is more pronounced for $\beta=1.60$.

Finally, it can be stressed that the single-phase flow statistics of the quantities considered in Figs. 8 and 9 are recovered at distances from the bubble surface comparable to $3 d_{b}$. Hydrodynamic interactions are therefore expected to be important only if two bubbles are located within approximately $6 d_{b}$ from each other. In the present setup, the separation distance between periodic neighbors is $11 d_{b}$ and the bubble can safely be considered as isolated.

Overall, our results suggest that finite-size bubbles preferentially sample downflow regions when $\beta \lesssim 1$, whereas they are primarily trapped in swirling zones when $\beta \gtrsim 1$, as is the case for point bubbles. The possible role played by the lift force in the biased sampling of the flow remains to be clarified. Finally, we stress that the conclusions drawn in this section are subject to caution: Since the effect of the bubble on the flow cannot be categorically disentangled from that of preferential sampling, the proposed interpretation of the conditional averages is not unequivocal. 


\section{CONCLUSION}

Interface-resolved numerical simulations of the rise of an isolated finite-size bubble in otherwise homogeneous isotropic turbulence were carried out for different values of the turbulence intensity $\beta$, defined as the root mean square of the liquid velocity fluctuations divided by the terminal velocity of the bubble in still liquid. These simulations were run over a time period long enough to allow a reasonable convergence of bubble Lagrangian and liquid Eulerian statistics.

The bubble kinematics was first characterized. Turbulence was found to drastically reduce its rise velocity, a property already known for microbubbles. Acceleration statistics display some features similar to those of fluid tracers, small inertial particles, and finite-size rigid objects. In particular, the bubble horizontal acceleration distribution is well fitted by a log-normal distribution. The PDF of the vertical component is negatively skewed, as a consequence of the preferential alignment of the bubble velocity with gravity and of the negative asymmetry of its longitudinal acceleration PDF. This latter property means that the bubble undergoes on average stronger deceleration than positive acceleration and had been previously evidenced for fluid tracers only (in that case it is related to the time irreversibility of turbulence). Then a physically relevant definition of the liquid flow experienced by the bubble, as it enters in usual models of the liquid acceleration force and of the lift force, was proposed. Our aim was not to propose a complete and accurate equation of motion of the bubble, but rather to propose some reasonable estimates of the liquid velocity and vorticity that enter in the definition of at least some of the typical forces known to act on inclusions suspended in simple flows. We think this approach opens alternative ways for the design of models of the dynamics of finite-size particles in turbulence, although it is still at a preliminary stage. Finally, the present simulations show that the behavior of a bubble much larger than the Kolmogorov scale is qualitatively similar to that of a small bubble in terms of preferential sampling of the turbulent flow. In particular, conditional averaging of the liquid flow in the bubble vicinity suggests that when $\beta \lesssim 1$ the bubble is more likely to reside in downflow regions of large extent, whereas when $\beta \gtrsim 1$ the bubble has a preference for swirling zones. Underlying mechanisms, however, still need to be elucidated.

The results presented in this paper were obtained at rather low Taylor Reynolds number and should be confirmed for higher values of it. However, previous investigations carried out at similar $\operatorname{Re}_{\lambda}$ have shed some light on the interaction between finite-size solid objects and turbulence $[16,17]$. The good qualitative agreement between our results and those known for small bubbles at $\operatorname{Re}_{\lambda}=O(100)$ is also very encouraging.

The above results were obtained using a turbulence forcing that consists in including a body force proportional to liquid velocity in the momentum conservation equation. As a further validation step, it would be desirable to reproduce the present simulations using a different forcing scheme. A possible alternative is the random forcing of [55] used in, e.g., the simulation of interface-resolved particle-laden flow [56].

\section{ACKNOWLEDGMENTS}

The authors would like to thank Peter Spelt for enlightening discussions and for helpful suggestions on this manuscript. This research was partially funded by the French research agency (Grant No. ANR-12-BS09-0011) and was performed using the HPC resources provided by GENCI-CINES and GENCI-IDRIS (Grant No. x20162b6893), PSMN (École Normale Supérieure de Lyon), P2CHPD (Université Claude Bernard Lyon 1), and PMCS2I (École Centrale de Lyon).

[1] L.-P. Wang and M. R. Maxey, The motion of microbubbles in a forced isotropic and homogeneous turbulence, Appl. Sci. Res. 51, 291 (1993). 
[2] M. R. Maxey, E. J. Chang, and L.-P. Wang, Simulation of interactions between microbubbles and turbulent flows, Appl. Mech. Rev. 47, S70 (1994).

[3] P. D. M. Spelt and A. Biesheuvel, On the motion of gas bubbles in homogeneous isotropic turbulence, J. Fluid Mech. 336, 221 (1997).

[4] I. M. Mazzitelli, D. Lohse, and F. Toschi, The effect of microbubbles on developed turbulence, Phys. Fluids 15, L5 (2003).

[5] I. M. Mazzitelli, D. Lohse, and F. Toschi, On the relevance of the lift force in bubbly turbulence, J. Fluid Mech. 488, 283 (2003).

[6] M. R. Snyder, O. M. Knio, J. Katz, and O. P. Le Maître, Statistical analysis of small bubble dynamics in isotropic turbulence, Phys. Fluids 19, 065108 (2007).

[7] S. Balachandar and J. K. Eaton, Turbulent dispersed multiphase flow, Annu. Rev. Fluid Mech. 42, 111 (2010).

[8] R. Volk, E. Calzavarini, E. Leveque, and J.-F. Pinton, Dynamics of inertial particles in a turbulent von Kármán flow, J. Fluid Mech. 668, 223 (2011).

[9] R. Zimmermann, Y. Gasteuil, M. Bourgoin, R. Volk, A. Pumir, and J.-F. Pinton, Rotational Intermittency and Turbulence Induced Lift Experienced by Large Particles in a Turbulent Flow, Phys. Rev. Lett. 106, 154501 (2011).

[10] G. Bellani and E. A. Variano, Slip velocity of large neutrally buoyant particles in turbulent flows, New J. Phys. 14, 125009 (2012).

[11] G. Bellani, M. L. Byron, A. G. Collignon, C. R. Meyer, and E. A. Variano, Shape effects on turbulent modulation by large nearly neutrally buoyant particles, J. Fluid Mech. 712, 41 (2012).

[12] S. Klein, M. Gibert, A. Bérut, and E. Bodenschatz, Simultaneous 3D measurement of the translation and rotation of finite-size particles and the flow field in a fully developed turbulent water flow, Meas. Sci. Technol. 24, 024006 (2013).

[13] V. Mathai, V. N. Prakash, J. Brons, C. Sun, and D. Lohse, Wake-Driven Dynamics of Finite-Sized Buoyant Spheres in Turbulence, Phys. Rev. Lett. 115, 124501 (2015).

[14] F. Ravelet, C. Colin, and F. Risso, On the dynamics and breakup of a bubble rising in a turbulent flow, Phys. Fluids 23, 1 (2011).

[15] V. N. Prakash, Y. Tagawa, E. Calzavarini, J. M. Mercado, F. Toschi, D. Lohse, and C. Sun, How gravity and size affect the acceleration statistics of bubbles in turbulence, New J. Phys. 14, 105017 (2012).

[16] A. Naso and A. Prosperetti, The interaction between a solid particle and a turbulent flow, New J. Phys. 12, 033040 (2010).

[17] H. Homann and J. Bec, Finite-size effects in the dynamics of neutrally buoyant particles in turbulent flow, J. Fluid Mech. 651, 81 (2010).

[18] M. Cisse, H. Homann, and J. Bec, Slipping motion of large neutrally buoyant particles in turbulence, J. Fluid Mech. 735, R1 (2013).

[19] A. Chouippe and M. Uhlmann, Forcing homogeneous turbulence in direct numerical simulation of particulate flow with interface resolution and gravity, Phys. Fluids 27, 123301 (2015).

[20] A. Merle, D. Legendre, and J. Magnaudet, Forces on a high-Reynolds-number spherical bubble in a turbulent flow, J. Fluid Mech. 532, 53 (2005).

[21] D. Legendre, A. Merle, and J. Magnaudet, Wake of a spherical bubble or a solid sphere set fixed in a turbulent environment, Phys. Fluids 18, 048102 (2006).

[22] J. Lu and G. Tryggvason, Numerical study of turbulent bubbly downflows in a vertical channel, Phys. Fluids 18, 103302 (2006).

[23] I. A. Bolotnov, K. E. Jansen, D. A. Drew, A. A. Oberai, R. T. Lahey, Jr., and M. Z. Podowski, Detached direct numerical simulations of turbulent two-phase bubbly channel flow, Int. J. Multiphase Flow 37, 647 (2011).

[24] J. Lu and G. Tryggvason, Dynamics of nearly spherical bubbles in a turbulent channel upflow, J. Fluid Mech. 732, 166 (2013).

[25] S. Dabiri, J. Lu, and G. Tryggvason, Transition between regimes of a vertical channel bubbly upflow due to bubble deformability, Phys. Fluids 25, 102110 (2013). 
[26] R. Mei, J. F. Klausner, and C. J. Lawrence, A note on the history force on a spherical bubble at finite Reynolds number, Phys. Fluids 6, 418 (1994).

[27] A. Chorin, Numerical solution of the Navier-Stokes equations, Math. Comput. 22, 745 (1968).

[28] M. Sussman, P. Smereka, and S. Osher, A level set approach for computing solutions to incompressible two-phase flow, J. Comput. Phys. 114, 146 (1994).

[29] V. Sabelnikov, A. Y. Ovsyannikov, and M. Gorokhovski, Modified level set equation and its numerical assessment, J. Comput. Phys. 278, 1 (2014).

[30] J. U. Brackbill, D. B. Kothe, and C. Zemach, A continuum method for modeling surface tension, J. Comput. Phys. 100, 335 (1992).

[31] G. Russo and P. Smereka, A remark on computing distance functions, J. Comput. Phys. 163, 51 (2000).

[32] M. Sussman and S. Uto, A computational study of the spreading of oil underneath a sheet of ice, CAM Report 98-32 (University of California, Los Angeles, 1998).

[33] A. Loisy, A. Naso, and P. D. M. Spelt, Buoyancy-driven bubbly flows: ordered and free rise at small and intermediate volume fraction, J. Fluid Mech. (to be published).

[34] Z. Amoura, V. Roig, F. Risso, and A.-M. Billet, Attenuation of the wake of a sphere in an intense incident turbulence with large length scales, Phys. Fluids 22, 055105 (2010).

[35] I. Eames, P. B. Johnson, V. Roig, and F. Risso, Effect of turbulence on the downstream velocity deficit of a rigid sphere, Phys. Fluids 23, 095103 (2011).

[36] T. S. Lundgren, Linearly forced isotropic turbulence, Annual Research Briefs (Center for Turbulence Research, Stanford, 2003), pp. 461-473.

[37] C. Rosales and C. Meneveau, Linear forcing in numerical simulations of isotropic turbulence: Physical space implementations and convergence properties, Phys. Fluids 17, 095106 (2005).

[38] B. Duret, G. Luret, J. Reveillon, T. Menard, A. Berlemont, and F. X. Demoulin, DNS analysis of turbulent mixing in two-phase flows, Int. J. Multiphase Flow 40, 93 (2012).

[39] J. C. R. Fung, Gravitational settling of particles and bubbles in homogeneous turbulence, J. Geophys. Res. 98, 20287 (1993).

[40] R. E. G. Poorte and A. Biesheuvel, Experiments on the motion of gas bubbles in turbulence generated by an active grid, J. Fluid Mech. 461, 127 (2002).

[41] A. Aliseda and J. C. Lasheras, Preferential concentration and rise velocity reduction of bubbles immersed in a homogeneous and isotropic turbulent flow, Phys. Fluids 23, 093301 (2011).

[42] F. Toschi and E. Bodenschatz, Lagrangian properties of particles in turbulence, Annu. Rev. Fluid Mech. 41, 375 (2009).

[43] N. M. Qureshi, U. Arrieta, C. Baudet, A. Cartellier, Y. Gagne, and M. Bourgoin, Acceleration statistics of inertial particles in turbulent flow, Eur. Phys. J. B 66, 531 (2008).

[44] N. Mordant, A. M. Crawford, and E. Bodenschatz, Three-Dimensional Structure of the Lagrangian Acceleration in Turbulent Flows, Phys. Rev. Lett. 93, 214501 (2004).

[45] N. M. Qureshi, M. Bourgoin, C. Baudet, A. Cartellier, and Y. Gagne, Turbulent Transport of Material Particles: An Experimental Study of Finite Size Effects, Phys. Rev. Lett. 99, 184502 (2007).

[46] E. Lévêque and A. Naso, Introduction of longitudinal and transverse Lagrangian velocity increments in homogeneous and isotropic turbulence, Europhys. Lett. 108, 54004 (2014).

[47] H. Xu, A. Pumir, G. Falkovich, E. Bodenschatz, M. Shats, H. Xia, N. Francois, and G. Boffetta, Flight-crash events in turbulence, Proc. Natl. Acad. Sci. USA 111, 7558 (2014).

[48] J. Magnaudet and I. Eames, The motion of high-Reynolds-number bubbles in inhomogeneous flows, Annu. Rev. Fluid Mech. 32, 659 (2000).

[49] D. Legendre and J. Magnaudet, The lift force on a spherical bubble in a viscous linear shear flow, J. Fluid Mech. 368, 81 (1998).

[50] M. Rastello, J.-L. Marié, and M. Lance, Drag and lift forces on clean spherical and ellipsoidal bubbles in a solid-body rotating flow, J. Fluid Mech. 682, 434 (2011).

[51] D. W. Moore, The boundary layer on a spherical gas bubble, J. Fluid Mech. 16, 161 (1963).

[52] K. J. Sene, J. C. R. Hunt, and N. H. Thomas, The role of coherent structures in bubble transport by turbulent shear flows, J. Fluid Mech. 259, 219 (1994). 


\section{AURORE LOISY AND AURORE NASO}

[53] B. J. Cantwell, Exact solution of a restricted Euler equation for the velocity gradient tensor, Phys. Fluids A 4, 782 (1992).

[54] A. Naso and A. Pumir, Scale dependence of the coarse-grained velocity derivative tensor structure in turbulence, Phys. Rev. E 72, 056318 (2005).

[55] K. Alvelius, Random forcing of three-dimensional homogeneous turbulence, Phys. Fluids 11, 1880 (1999).

[56] A. Ten Cate, J. J. Derksen, L. M. Portela, and H. E. A. van den Akker, Fully resolved simulations of colliding monodisperse spheres in forced isotropic turbulence, J. Fluid Mech. 519, 233 (2004). 